\title{
Tensor extension of the Poincaré algebra
}

\author{
Dmitrij V. Soroka* and Vyacheslav A. Soroka ${ }^{\dagger}$ \\ Kharkov Institute of Physics and Technology, 61108 Kharkov, Ukraine
}

\begin{abstract}
A tensor extension of the Poincaré algebra is proposed for the arbitrary dimensions. Casimir operators of the extension are constructed. A possible supersymmetric generalization of this extension is also found in the dimensions $D=2,3,4$.
\end{abstract}

$P A C S: 11.30 . \mathrm{Cp} ; 11.30 . \mathrm{Pb}$

Keywords: Poincaré algebra, Tensor, Extension, Casimir operators, Supersymmetry

\footnotetext{
${ }^{*}$ E-mail: dsoroka@kipt.kharkov.ua
}

${ }^{\dagger}$ E-mail: vsoroka@kipt.kharkov.ua 


\section{Introduction}

There are many examples for the tensor (semi-central) extensions of the super-Poincaré algebra (see, for example, 11, 2, 3, 4, 15, 6, 7]). However, there also exists the tensor extension of the Poincaré algebra itself. In the present paper we give the example of such an extension with the help of the second rank tensor generator. A consideration of such an extension may have a sense, since it is homomorphic to the Poincaré algebra. Moreover, the contraction of the extended algebra leads also to the Poincaré algebra. It is interesting enough that the momentum square Casimir operator for the Poincaré algebra under this extension ceases to be the Casimir operator and it is generalized by adding the term containing linearly the angular momentum ${ }^{1}$. Due to this fact, an irreducible representation of the extended algebra ${ }^{2}$ has to contain the fields of the different masses. This extension with non-commuting momenta has also something in common with the ideas of the papers [9, 10, 11] and with the non-commutative geometry idea 12. It is also shown that for the dimensions $D=2,3,4$ the extended Poincaré algebra allows a supersymmetric generalization.

\section{Extension of the Poincaré algebra}

The Poincaré algebra for the components of the rotations $M_{a b}$ and translations $P_{a}$ in $D$ dimensions

$$
\begin{gathered}
{\left[M_{a b}, M_{c d}\right]=\left(g_{a d} M_{b c}+g_{b c} M_{a d}\right)-(c \leftrightarrow d),} \\
{\left[M_{a b}, P_{c}\right]=g_{b c} P_{a}-g_{a c} P_{b},} \\
{\left[P_{a}, P_{b}\right]=0}
\end{gathered}
$$

can be extended with the help of the tensor semi-central generator $Z_{a b}$ in the following way:

$$
\begin{gathered}
{\left[M_{a b}, M_{c d}\right]=\left(g_{a d} M_{b c}+g_{b c} M_{a d}\right)-(c \leftrightarrow d),} \\
{\left[M_{a b}, P_{c}\right]=g_{b c} P_{a}-g_{a c} P_{b},} \\
{\left[P_{a}, P_{b}\right]=Z_{a b},} \\
{\left[M_{a b}, Z_{c d}\right]=\left(g_{a d} Z_{b c}+g_{b c} Z_{a d}\right)-(c \leftrightarrow d),} \\
{\left[P_{a}, Z_{b c}\right]=0,}
\end{gathered}
$$

\footnotetext{
${ }^{1}$ Note that this reminds the relation for the Regge trajectory, which connects the mass square with the angular momentum.

${ }^{2}$ Concerning the irreducible unitary representations of the extended Poincaré group in $(1+1)$ dimensions see, for example, [8].
} 


$$
\left[Z_{a b}, Z_{c d}\right]=0
$$

By taking a set of the generators $Z_{a b}$ as a homomorphism kernel, we obtain that the extended Poincaré algebra (2.2) is homomorphic to the usual Poincaré algebra (2.1). Under a contraction $Z_{a b} \rightarrow 0$ the algebra (2.2) also goes to the Poincaré algebra (2.1).

Casimir operators of the extended Poincaré algebra are

$$
\begin{aligned}
& Z_{a_{1} a_{2}} Z^{a_{2} a_{3}} \cdots Z_{a_{2 k-1} a_{2 k}} Z^{a_{2 k} a_{1}}, \quad(k=1,2, \ldots) \\
& P^{a_{1}} Z_{a_{1} a_{2}} Z^{a_{2} a_{3}} \quad \cdots \quad Z_{a_{2 k-1} a_{2 k}} Z^{a_{2 k} a_{2 k+1}} P_{a_{2 k+1}} \\
& +Z^{a a_{1}} Z_{a_{1} a_{2}} \cdots Z_{a_{2 k-1} a_{2 k}} Z^{a_{2 k} a_{2 k+1}} M_{a_{2 k+1} a}, \quad(k=0,1,2, \ldots) \\
& \epsilon^{a b c d} Z_{a b} Z_{c d}
\end{aligned}
$$

where $\epsilon^{a b c d}, \epsilon^{0123}=1$ is the totally antisymmetric Levi-Civita tensor. In particular, there is a Casimir operator generalizing the momentum square

$$
P^{a} P_{a}+Z^{a b} M_{b a}
$$

which indicates that an irreducible representation of the extended algebra contains the fields having the different masses. Note that for the extended algebra there is no generalization of the Pauli-Lubanski vector of the Poincaré algebra. The expressions (2.3) and (2.4) for the Casimir operators are valid for the extended Poincaré algebra (2.2) in the arbitrary dimensions $D$, but the expression (2.5) is only true for $D=4$.

Note that in the case of the extended two-dimensional Poincaré algebra the Casimir operators (2.3) and (2.4) can be expressed

$$
\begin{aligned}
& Z_{a_{1} a_{2}} Z^{a_{2} a_{3}} \cdots Z_{a_{2 k-1} a_{2 k}} Z^{a_{2 k} a_{1}}=2 Z^{2 k} \\
& P^{a_{1}} Z_{a_{1} a_{2}} Z^{a_{2} a_{3}} \quad \cdots \quad Z_{a_{2 k-1} a_{2 k}} Z^{a_{2 k} a_{2 k+1}} P_{a_{2 k+1}} \\
& +Z^{a a_{1}} Z_{a_{1} a_{2}} \quad \cdots \quad Z_{a_{2 k-1} a_{2 k}} Z^{a_{2 k} a_{2 k+1}} M_{a_{2 k+1} a}=Z^{2 k}\left(P^{a} P_{a}+Z^{a b} M_{b a}\right)
\end{aligned}
$$

as degrees of the following generating Casimir operators:

$$
\begin{gathered}
Z=\frac{1}{2} \epsilon^{a b} Z_{a b}, \\
P^{a} P_{a}+Z^{a b} M_{b a},
\end{gathered}
$$

where $\epsilon^{a b}=-\epsilon^{b a}, \epsilon^{01}=1$ is the completely antisymmetric two-dimensional Levi-Civita tensor. In the case of the extended three-dimensional Poincaré algebra these Casimir operators can be expressed

$$
Z_{a_{1} a_{2}} Z^{a_{2} a_{3}} \cdots Z_{a_{2 k-1} a_{2 k}} Z^{a_{2 k} a_{1}}=2\left(Z^{a} Z_{a}\right)^{k}
$$




$$
\begin{array}{rll}
P^{a_{1}} Z_{a_{1} a_{2}} Z^{a_{2} a_{3}} & \cdots & Z_{a_{2 k-1} a_{2 k}} Z^{a_{2 k} a_{2 k+1}} P_{a_{2 k+1}} \\
+Z^{a a_{1}} Z_{a_{1} a_{2}} & \cdots & Z_{a_{2 k-1} a_{2 k}} Z^{a_{2 k} a_{2 k+1}} M_{a_{2 k+1} a} \\
& =\left(Z^{a} Z_{a}\right)^{k}\left(P^{a} P_{a}+Z^{a b} M_{b a}\right)-\left(Z^{a} Z_{a}\right)^{k-1}\left(P^{a} Z_{a}\right)^{2}
\end{array}
$$

in terms of the following generating Casimir operators:

$$
\begin{gathered}
Z^{a} Z_{a}, \\
P^{a} P_{a}+Z^{a b} M_{b a}, \\
P^{a} Z_{a},
\end{gathered}
$$

where

$$
Z^{a}=\frac{1}{2} \epsilon^{a b c} Z_{b c}
$$

and $\epsilon^{a b c}, \epsilon^{012}=1$ is the totally antisymmetric three-dimensional Levi-Civita tensor. In the case of the extended $D$-dimensional $(D \geq 4)$ Poincaré algebra the Casimir operators (2.3) and (2.4) can not be expressed in terms of the finite number of the generating Casimir operators.

Generators of the left shifts, acting on the function $f(y)$ with a group element $G$,

$$
[T(G) f](y)=f\left(G^{-1} y\right), \quad y=\left(x^{a}, z^{a b}\right)
$$

have the form

$$
\begin{gathered}
P_{a}=-\left(\frac{\partial}{\partial x^{a}}+\frac{1}{2} x^{b} \frac{\partial}{\partial z^{a b}}\right), \\
Z_{a b}=-\frac{\partial}{\partial z^{a b}} \\
M_{a b}=x_{a} \frac{\partial}{\partial x^{b}}-x_{b} \frac{\partial}{\partial x^{a}}+z_{a}^{c} \frac{\partial}{\partial z^{b c}}-z_{b}^{c} \frac{\partial}{\partial z^{a c}}+S_{a b},
\end{gathered}
$$

where coordinates $x^{a}$ correspond to the translation generators $P_{a}$, coordinates $z^{a b}$ correspond to the generators $Z_{a b}$ and $S_{a b}$ is a spin operator.

On the other hand, generators of the right shifts

$$
[T(G) f](y)=f(y G)
$$

have the form

$$
D \stackrel{\text { def }}{=} P_{a}^{r}=\frac{\partial}{\partial x^{a}}-\frac{1}{2} x^{b} \frac{\partial}{\partial z^{a b}},
$$




$$
Z_{a b}^{r}=-Z_{a b}=\frac{\partial}{\partial z^{a b}}
$$

Note that the algebra

$$
\begin{gathered}
{\left[M_{a b}, M_{c d}\right]=\left(g_{a d} M_{b c}+g_{b c} M_{a d}\right)-(c \leftrightarrow d),} \\
{\left[M_{a b}, P_{c}\right]=g_{b c} P_{a}-g_{a c} P_{b},} \\
{\left[M_{a b}, D_{c}\right]=g_{b c} D_{a}-g_{a c} D_{b},} \\
{\left[P_{a}, P_{b}\right]=Z_{a b},} \\
{\left[D_{a}, D_{b}\right]=-Z_{a b},} \\
{\left[P_{a}, D_{b}\right]=0,} \\
{\left[g_{a b}, Z_{c d}\right]=\left[g_{b c}+g_{b c} Z_{a d}\right)-(c \leftrightarrow d),} \\
{\left[P_{a}, Z_{b c}\right]=0,} \\
{\left[D_{a}, Z_{b c}\right]=0,} \\
{\left[Z_{c d}\right]=0,}
\end{gathered}
$$

formed by the generators $M_{a b}, P_{a}, D_{a}$ and $Z_{a b}$, has as Casimir operators the operators (2.3) and the following operators:

$$
\begin{aligned}
& (P-D)^{a_{1}} Z_{a_{1} a_{2}} Z^{a_{2} a_{3}} \quad \ldots \quad Z_{a_{2 k-1} a_{2 k}} Z^{a_{2 k} a_{2 k+1}}(P+D)_{a_{2 k+1}} \\
& +Z^{a a_{1}} Z_{a_{1} a_{2}} \quad \cdots \quad Z_{a_{2 k-1} a_{2 k}} Z^{a_{2 k} a_{2 k+1}} M_{a_{2 k+1} a}, \quad(k=0,1,2, \ldots) .
\end{aligned}
$$

\section{Supersymmetric generalization}

The extended Poincaré algebra (2.2) admits the following supersymmetric generalization:

$$
\begin{gathered}
\left\{Q_{\alpha}, Q_{\beta}\right\}=-d\left(\sigma^{a b} C\right)_{\alpha \beta} Z_{a b}, \\
{\left[M_{a b}, Q_{\alpha}\right]=-\left(\sigma_{a b} Q\right)_{\alpha},} \\
{\left[P_{a}, Q_{\alpha}\right]=0,}
\end{gathered}
$$




$$
\left[Z_{a b}, Q_{\alpha}\right]=0
$$

with the help of the super-translation generators

$$
Q_{\alpha}=-\left[\frac{\partial}{\partial \bar{\theta}^{\alpha}}+\frac{d}{2}\left(\sigma^{a b} \theta\right)_{\alpha} \frac{\partial}{\partial z^{a b}}\right],
$$

where $\theta=C \bar{\theta}$ is a Majorana Grassmann spinor, $C$ is a charge conjugation matrix, $d$ is some constant and $\sigma_{a b}=\frac{1}{4}\left[\gamma_{a}, \gamma_{b}\right]$.

The rotation generators acquire the terms depending on the Grassmann variables $\theta_{\alpha}$

$$
M_{a b}=x_{a} \frac{\partial}{\partial x^{b}}-x_{b} \frac{\partial}{\partial x^{a}}+z_{a}^{c} \frac{\partial}{\partial z^{b c}}-z_{b}^{c} \frac{\partial}{\partial z^{a c}}-\left(\sigma_{a b} \theta\right)_{\alpha} \frac{\partial}{\partial \theta_{\alpha}}+S_{a b},
$$

whereas the expressions for the translations $P_{a}$ and tensor generator $Z_{a b}$ remain unchanged.

The validity of the Jacobi identities

$$
\left[P_{a},\left\{Q_{\alpha}, Q_{\beta}\right\}\right]=\left\{Q_{\alpha},\left[P_{a}, Q_{\beta}\right]\right\}+\left\{Q_{\beta},\left[P_{a}, Q_{\alpha}\right]\right\}
$$

and

$$
\left[M_{a b},\left\{Q_{\alpha}, Q_{\beta}\right\}\right]=\left\{Q_{\alpha},\left[M_{a b}, Q_{\beta}\right]\right\}+\left\{Q_{\beta},\left[M_{a b}, Q_{\alpha}\right]\right\}
$$

for the supersymmetric generalization of the extended Poincaré algebra (2.2) verified for the dimensions $D=2,3,4$ with the use of the symmetry properties of the matrices $C$ and $\gamma_{a} C$ and the relations (A.1) - A.3 of the Appendix.

One of the generating Casimir operator in the dimensions $D=2,3$ is generalized into the following form:

$$
P^{a} P_{a}+Z^{a b} M_{b a}-\frac{1}{2 d} Q_{\alpha}\left(C^{-1}\right)^{\alpha \beta} Q_{\beta},
$$

while the form of the rest generating Casimir operators in these dimensions are not changed. Note that in the case $D=3$ there is also the following Casimir operator:

$$
Z^{a} Q_{\alpha}\left(C^{-1} \gamma_{a}\right)^{\alpha \beta} Q_{\beta}
$$

One of the simplest Casimir operator (2.6) in $D=4$ is also generalized into the form (3.1). The supersymmetric generalization of the more complicated Casimir operators in the four-dimensional case has the following structure:

$$
\begin{aligned}
P^{a} Z_{a b} Z^{b c} P_{c}+Z^{a b} Z_{b c} Z^{c d} & M_{d a}+\frac{2}{5 d} Q_{\alpha}\left(C^{-1} \sigma^{a b} Z_{a b} \sigma^{c d} Z_{c d}\right)^{\alpha \beta} Q_{\beta}+\frac{1}{2 d} Z^{a b} Z_{a b} Q_{\alpha}\left(C^{-1}\right)^{\alpha \beta} Q_{\beta}, \\
P^{a} Z_{a b} Z^{b c} Z_{c d} Z^{d e} P_{e} & +Z^{a b} Z_{b c} Z^{c d} Z_{d e} Z^{e f} M_{f a} \\
& +\frac{2}{5 d} Q_{\alpha}\left[C^{-1} \sigma^{a b} Z_{a b} \sigma^{c d}\left(Z_{c e} Z^{e f} Z_{f d}+\frac{3}{10} Z^{g h} Z_{h g} Z_{c d}\right)\right]^{\alpha \beta} Q_{\beta} \\
& -\frac{1}{20 d}\left[7 Z_{a b} Z^{b c} Z_{c d} Z^{d a}+3\left(Z^{e f} Z_{f e}\right)^{2}\right] Q_{\alpha}\left(C^{-1}\right)^{\alpha \beta} Q_{\beta} .
\end{aligned}
$$


An algorithm for the construction of the supersymmetric generalization of the Casimir operators (2.4) is obvious and based on the use of the following commutation relations:

$$
\begin{gathered}
{\left[\frac{1}{2 d} Q_{\alpha}\left(C^{-1}\right)^{\alpha \beta} Q_{\beta}, Q_{\gamma}\right]=Z^{a b}\left(\sigma_{a b} Q\right)_{\gamma}} \\
{\left[\frac{2}{5 d} Q_{\alpha}\left(C^{-1} \sigma^{a b} Z_{a b} \sigma^{c d} \tilde{Z}_{c d}\right)^{\alpha \beta} Q_{\beta}, Q_{\gamma}\right]=\left(Z^{a b} Z_{b c} \tilde{Z}^{c d}\right.} \\
+\frac{7}{10} Z_{b c} \tilde{Z}^{c b} Z^{a d} \\
\left.+\frac{3}{10} Z_{b c} Z^{c b} \tilde{Z}^{a d}\right)\left(\sigma_{a d} Q\right)_{\gamma}
\end{gathered}
$$

where

$$
\tilde{Z}^{a b}=Z^{a a_{1}} Z_{a_{1} a_{2}} \cdots Z_{a_{2 k-1} a_{2 k}} Z^{a_{2 k} b}, \quad(k=0,1, \ldots) .
$$

\section{Conclusion}

Thus, in the present paper we proposed the extension of the Poincaré algebra with the help of the second rank tensor generator. Casimir operators for the extended algebra are constructed. The form of the Casimir operators indicate that an irreducible representation of the extended algebra contains the fields with the different masses. A consideration is performed for the arbitrary dimensions $D$. A possible supersymmetric generalization of the extended Poincaré algebra is also given for the particular cases with the dimensions $D=2,3,4$.

It would be interesting to find the spectra of the Casimir operators and to construct the models based on the extended Poincaré algebra. The work in this direction is in progress.

\section{Acknowledgments}

One of the authors (V.A.S.) would like to thank B.A. Dubrovin for useful discussions. V.A.S. is sincerely grateful to L. Bonora for fruitful discussions and for kind hospitality at SISSA/ISAS (Trieste), where the main part of this work has been performed.

\section{A Appendix}

As a real (Majorana) representation for the two-dimensional $\gamma$-matrices and charge conjugation matrix $C$ we adopt

$$
\begin{gathered}
\gamma^{0}=C=-C^{T}=-i \sigma_{2}, \quad \gamma^{1}=\sigma_{1}, \quad \gamma_{5}=\frac{1}{2} \epsilon^{a b} \gamma_{a} \gamma_{b}=\sigma_{3} \\
\left\{\gamma_{a}, \gamma_{b}\right\}=2 g_{a b}, \quad g_{11}=-g_{00}=1, \quad C^{-1} \gamma_{a} C=-\gamma_{a}^{T}
\end{gathered}
$$

where $\sigma_{i}$ are Pauli matrices. The matrices $\gamma_{a}$ satisfy the relations

$$
\gamma_{a} \gamma_{5}=\epsilon_{a b} \gamma^{b}, \quad \gamma_{a} \gamma_{b}=g_{a b}-\epsilon_{a b} \gamma_{5}
$$


For the Majorana three-dimensional $\gamma$-matrices and charge conjugation matrix $C$ we take

$$
\begin{gathered}
\gamma^{0}=C=-C^{T}=-i \sigma_{2}, \quad \gamma^{1}=\sigma_{1}, \quad \gamma^{2}=\sigma_{3} ; \\
\left\{\gamma_{a}, \gamma_{b}\right\}=2 g_{a b}, \quad g_{11}=g_{22}=-g_{00}=1, \quad C^{-1} \gamma_{a} C=-\gamma_{a}^{T},
\end{gathered}
$$

The matrices $\gamma_{a}$ obey the relations

$$
\gamma_{a} \gamma_{b}=g_{a b}-\epsilon_{a b c} \gamma^{c} .
$$

At last, the real four-dimensional $\gamma$-matrices and matrix $C$ are

$$
\begin{gathered}
\gamma^{0}=C=-C^{T}=-i\left(\begin{array}{cc}
0 & \sigma_{2} \\
\sigma_{2} & 0
\end{array}\right), \quad \gamma^{1}=\left(\begin{array}{cc}
\sigma_{3} & 0 \\
0 & \sigma_{3}
\end{array}\right), \\
\gamma^{2}=i\left(\begin{array}{cc}
0 & \sigma_{2} \\
-\sigma_{2} & 0
\end{array}\right), \quad \gamma^{3}=-\left(\begin{array}{cc}
\sigma_{1} & 0 \\
0 & \sigma_{1}
\end{array}\right), \\
\left\{\gamma_{a}, \gamma_{b}\right\}=2 g_{a b}, \quad g_{11}=g_{22}=g_{33}=-g_{00}=1, \quad C^{-1} \gamma_{a} C=-\gamma_{a}^{T}, \quad \gamma_{5}=\frac{1}{4} \epsilon^{a b c d} \gamma_{a} \gamma_{b} \gamma_{c} \gamma_{d} .
\end{gathered}
$$

The matrices $\gamma_{a}$ and $\sigma_{a b}$ meet the relations

$$
\begin{gathered}
\gamma_{a} \sigma_{b c}=\frac{1}{2} \epsilon_{a b c d} \gamma^{d} \gamma_{5}+\frac{1}{2}\left(\gamma_{c} g_{a b}-\gamma_{b} g_{a c}\right), \quad \sigma_{a b} \gamma_{c}=\frac{1}{2} \epsilon_{a b c d} \gamma^{d} \gamma_{5}+\frac{1}{2}\left(\gamma_{a} g_{b c}-\gamma_{b} g_{a c}\right), \\
\sigma_{a b} \sigma_{c d}=\frac{1}{4}\left(g_{a d} g_{b c}-g_{a c} g_{b d}-\epsilon_{a b c d} \gamma_{5}\right)+\frac{1}{2}\left(\sigma_{a d} g_{b c}+\sigma_{b c} g_{a d}-\sigma_{a c} g_{b d}-\sigma_{b d} g_{a c}\right) .
\end{gathered}
$$

\section{References}

[1] R. D'Auria and P. Fre, Nucl. Phys. B201 (1982) 101.

[2] J. van Holten and A. van Proyen, J. Physics A15 (1982) 3763.

[3] P.A. Zizzi, Phys. Lett. 149B (1984) 333.

[4] J. Huges and J. Polchinski, Nucl. Phys. B278 (1986) 147.

[5] D.V. Volkov, V.A. Soroka and V.I. Tkach, Ukr. Fiz. Zh. (Russ. Ed.) 32 (1987) 1622.

[6] J.A. de Azcarraga, J.P. Gauntlett, J.M. Izquierdo and P.K. Townsend, Phys. Rev. Lett. 63 (1989) 2443.

[7] M. Duff and K.S. Stelle, Phys. Lett. 253B (1991) 113.

[8] R.O. de Mello and V.O. Rivelles, J. Math. Phys. 45 (2004) 1156.

[9] H.S. Snyder, Phys. Rev. 71 (1947) 38.

[10] C.N. Yang, Phys. Rev. 72 (1947) 874.

[11] V.V. Khruschev and A.N. Leznov, Grav. Cosmol. 9 (2003) 159.

[12] A. Connes, Publ. math. Inst. hautes étud. sci. 62 (1985) 41. 